\title{
Quality assurance of genetic laboratories and the EBTNA practice certification, a simple standardization assurance system for a laboratory network
}

\author{
Vincenza Precone ${ }^{1}$, Munis Dundar ${ }^{2}$, Tommaso Beccari ${ }^{3}$, Eda Tahir Turanli ${ }^{4}$, Stefano Cecchin ${ }^{5,6}$, \\ Giuseppe Marceddu ${ }^{1}$, Elena Manara ${ }^{1,5}$ and Matteo Bertelli ${ }^{1,5 *}$
}

\begin{abstract}
Analytical laboratory results greatly influence medical diagnosis, about $70 \%$ of medical decisions are based on laboratory results. Quality assurance and quality control are designed to detect and correct errors in a laboratory's analytical process to ensure both the reliability and accuracy of test results. Unreliable performance can result in misdiagnosis and delayed treatment. Furthermore, improved quality guarantees increased productivity at a lower cost. Quality assurance programmes include internal quality control, external quality assessment, proficiency surveillance and standardization. It is necessary to try to ensure compliance with the requirements of the standards at all levels of the process. The sources of these standards are the International Standards Organization (ISO), national standards bodies, guidelines from professional organisations, accreditation bodies and governmental regulations. Laboratory networks increase the performance of laboratories in support of diagnostic screening programme. It is essential that genetic laboratories of a network have procedures underpinned by a robust quality assurance system to minimize errors and to reassure the clinicians and the patients that international standards are being met. This article provides an overview of the bases of quality assurance and its importance in genetic tests and it reports the EBTNA quality assurance system which is a clear and simple system available for access to adequate standardization of a genetic laboratory's network.
\end{abstract}

Keywords: Quality assurance, ISO, EBTNA Quality System, laboratory network, genetic tests

${ }^{1}$ MAGI Euregio, Bolzano, Italy

${ }^{2}$ Department of Medical Genetics, Erciyes University Medical School, Kayseri, Turkey

${ }^{3}$ Department of Pharmaceutical Sciences, University of Perugia, Perugia, Italy

${ }^{4}$ Department of Molecular Biology and Genetics, Istanbul Technical University, Istanbul, Turkey

${ }^{5}$ EBTNA-LAB, Rovereto, Italy

${ }^{6}$ MAGI's lab, Rovereto, Italy

${ }^{*}$ Corresponding author: M. Bertelli

E-mail: info@assomagi.org

DOI: 10.2478/ebtj-2018-0052

(c) 2018 Authors. This work was licensed under the Creative Commons AttributionNonCommercial-NoDerivs 3.0 License.

\section{Introduction}

Laboratory networks are an important component of disease genetic screening systems because they provide accurate and timely diagnosis, but they need a common and standardized quality system to guarantee safe and uniform results. Genetic laboratories use a wide variety of technologies and new tests are continuously being introduced (www.genetests.org). Therefore, new predictive genetic tests need to be available with an appropriate assessment of their validity, benefits and utility (1). The optimal quality management of the analysis and the interpretation of genetic tests are complex and important, because the result is used for carrier identification, presymptomatic identification of individual risk and prediction of therapy responses (2). The reliability of results of laboratory analysis is fundamental because about $70 \%$ of clinical decisions are based on laboratory outcomes (3). It is necessary for genetic laboratories to have a quality system that allows to monitor all aspects: technical (pre-analytical, analytical and post-analytical processes), communicative (comprehensible messages and timely responses) and economical $(4,5)$. A rapid increase in the number of DNA based diagnostic tests of genetic diseases has given rise to the need of national and international genetic laboratory networks. There are many challenges facing the development and maintenance of laboratory networks, but the rewards provided by a laboratory network are as numerous (6). The main network 
of laboratories is GENDIA, that is an international network consisting of more than 100 genetic laboratories. The European Biotechnology Thematic Network Association (EBTNA) provides a European laboratory network similar to the GENDIA laboratory network but with a certified standard of quality. Unlike GENDIA, which provides a quality system that complies with the law of the countries of affiliated laboratories but not a quality system common to all the components of the laboratory network, EBTNA has developed a simplified quality system for the laboratory network to have a common quality system in according to the international standards that guarantees transparency and uniformity of results. The EBTNA laboratory provide genotyped samples to laboratories affiliated by paying a fee only after the adhesion of the laboratories to the specific and simplified EBTNA quality system. It is expected that all the laboratories involved will have to demonstrate a quality standard and an implementation of the informatic structures and the process platforms before being part of the laboratory network.

\section{Quality management system of analytical}

laboratories: Internal quality assurance, external quality assessment and accreditation.

Quality assurance (QA) is crucial to maintaining the highest quality standards in genetic testing laboratories. The QA is based on two tools: process control and quality statistical control (www.bayes.it). In a clinical laboratory the process control coincides with the control of sources of analytic variability, so that it has an accurate end result. The outcome of a laboratory test results from a "variability" factor related both to laboratory activities (preanalytical, analytic and post analytical variability) and to the subject itself (inter-individual and intra-individual biological variability) that result in a total variability that causes each laboratory data to be affected by a greater or lesser extent of error. The preanalytical phase comprises test selection and specimen collection, processing, handling, and delivery to the testing site; the analytic phase includes selection of test methods, performance of test procedures, monitoring and verification of the accuracy and reliability of test results, and documentation of test findings; The postanalytical phase includes reporting test results and archiving records, reports, and tested specimens (7). Errors are very likely to emerge during the pre-analytical phase and post-analytical phases (8). The statistical approach is helpful for the QA, it is based on the fact that the measurement errors are distributed according to the Gaussian model, therefore, once a maximum error tolerance limit has been established, the error can be detected and reported within the tolerance limits (9). The automated analysers are capable of producing multiple results in a short time through the incorporation of robotics and bioinformatics. However, statistical quality control is difficult to monitor due to the large number of samples analysed (10). Furthermore, the implement of new technologies, e.g. Next Generation Sequencing, requires an optimal standardization for the reduction of errors in medical laboratories $(11,12)$.

The analytical quality management of a laboratory is carried out by adopting different types of QA programs, an internal quality assessment (IQA) and an external quality assessment (EQA) (13). The World Health Organization (WHO) defines IQC as "the set of procedures undertaken by the staff of a laboratory for continuously assessing laboratory work and the emergent results, in order to decide whether they are reliable enough to be released". The IQA provide to maintain a high degree of confidence in test accuracy, but not necessarily of precision; its procedures include sample input, management, test, interpretation of results, and report output. IQA has a dual objective: controlling the analytical performance of the method and verifying the medium and long-term stability of the analytical method. Intra-laboratory quality control means checking genetic test results by using algorithms for measuring control materials, mainly to assess their reproducibility. An important component in the organization of the internal quality control is the selection of adequate control material. Multicentral management of an "extended" internal quality assessment to affiliated laboratories is very important and it can be facilitated by the use of software that allows a real-time update of its data management and of that other laboratories. This control system is the one that mainly consents for continuous monitoring because the widespread use of control materials and their daily use provide greater data availability, both as a number and as frequency distribution, which allows comparison of results between different labs that use the same control materials $(13,14)$. EQA is defined by WHO as "a system of objectively checking laboratory results by means of an external agency. The checking is necessarily retrospective, and the comparison of a given laboratory's performance on a certain day with that of other laboratories cannot be notified to the laboratory until some time later. The main objective of EQA is not to bring about day-to-day consistency, but to establish interlaboratory compatibility" $(13,15)$. The EQA comparing test results with those obtained from different laboratories or recognized standards is a key element for measuring the reliability of test results (16). EQA programs allow a periodic and retrospective estimate of the total error. The laboratories participating in the program receive a report with information processed by the provider. EQA programs are not intended to improve the performance of a laboratory medicine service, but are an index to measure effectiveness (15). Grading report content is more important than scoring genotypes, and best practice guidelines provide an important framework for this process. The molecular genetic test reports must be accurate and complete with all the information to enable effective decision-making by doctors and patients (17). It is important to follow-up EQA results and discuss the reports in laboratory meetings and preventive and corrective actions should be documented. EQA provides continuous education and training for laboratories (18). The accreditation of the genetic laboratories by national or international accreditation bodies such as ILAC (International Laboratory Accreditation Cooperation) or EA (European Cooperation for Accreditation) against international standards, such as ISO (International Organization for Standardization) is important. In 
particular, adherence and "good conduct" to an EQA program are key and qualifying elements for all professional certification and accreditation programs (19).

ISO accredited certification: The gold standard for the accreditation of medical laboratories.

The concept of quality as "total quality" and "quality management" finds its utmost expression with the ISO 9000, a set of standards defining a "quality system" model designed as a tool of character, organizational and managerial focused on monitoring and controlling processes that have a direct influence on the quality of the service provided (20). The ISO has developed an international standard for the accreditation of testing laboratories in general (ISO 17025) and one specifically for medical laboratories (ISO 15189) $(21,22)$. The genetic laboratories make use of this accreditation system. The rational of the IS0 15189 is based on the following phases: Description of process and identification of critical phases; detection of the verification steps of the analysis process and development of a sample traceability system in the analysis process; development of a training plan, a calibration and maintenance plan, internal and external quality control systems, and analysis of results with possible implementation or corrective action plans, indication of procedures, protocols and modules. More precisely, the ISO 15189 accreditation standard covers two sections: management requirements and technical requirements of QA. Management elements include document control, identification of non-conformities, implementing of corrective and preventive actions, action plans, performance of internal audit and management review, resolution of complaints, evaluation of external services, suppliers, contracts and referral laboratories. Technical elements comprise personnel and training, accommodation, equipment, validation and assuring quality of examination procedures by IQC, EQA, maintenance and calibration. The fundamental principles of ISO 15189 are: The laboratory management has a responsibility to manage; the laboratory must know who are its users and meet their requirements and the physical laboratory should not interfere with laboratory workers or laboratory samples $(22,23)$.

Genetic laboratories must have various elements of quality assurance to maintain a consistently high standard of performance in according to ISO 15189 (24). The principal key systems for implementation of quality management are:

- Accommodation and environmental conditions: The laboratory shall have a space allocated for the performance of its work that is designed to ensure the quality, safety and efficacy of the service provided to the users and to the health and safety of laboratory personnel, patients and visitors (25).

- Use validated diagnostic tests: Laboratories must show that used diagnostic tests that can consistently deliver the correct genotype under standard working conditions. Scientists should publish their validation work in specialized journals. A very important component is the estimation of "accuracy" for quantitative and qualitative tests. The components of accuracy for qualitative tests are sensitivity that is a measure of how correctly the test detects positive results and specificity which describes how correctly negatives are detected. Accuracy involves precision and authenticity for quantitative and semiquantitative tests. The precision includes the repeatability, reproducibility, intermediate precision and robustness and the accuracy is the closeness of agreement with a reference value (26). A standardized framework for the validation and verification of clinical molecular genetic tests was developed from EuroGentest (27).

- References materials: To verify that the result of an assay is correct is necessary to run positive control samples. Reference samples are positive controls supplied with commercial diagnostic kits, positive controls developed from the laboratory verified by a second assay method or controls obtained from another laboratory (28). The European Commission has developed a range of certified reference materials for diagnostic testing which are refereed in general guidelines (29).

- Document control: Document control is the periodic process that comprises creation, approbation, distribution, review, revision, and archiviation of the QA documents (30). The document control must be uniquely identifiable and signalized with a version number, a date of issue and the name or signature of the authorizing person (31).

- Internal audit: The internal audit is a means to improve the system led by laboratory itself. In an internal meeting the auditor should combine effectively interviews, document control, observations and cross-checks. The preparation of an internal audit should include the identification of all reference documents, for example SOPs and standards. Internal auditing consists of three phases: preparation, execution, and reporting and follow-up. The internal audit must be conducted once every 12 months in according to ISO 15189 (32).

- Identification of non-conformities: A non-conformity is a failure to meet a requirement of the quality management system or the relevant standard. In presence of non-conformities, corrective and preventive actions should be implemented to eliminate it. Reporting non-conformities, developing action plans and re-evaluating is also known as the Plan-Do-CheckAct (PDCA) circle which repeatedly performs in pursuit of continuous improvement $(33,34)$.

- Management review: In ISO 15189 is reported that "laboratory management shall review the quality management system to ensure continuing suitability and effectiveness in support of patient care and to introduce any necessary changes or improvements. The results of the review shall be incorporated into a plan that includes goals, objectives and action plans (35). Laboratory management must apply quality indicators for systematically monitoring and evaluating laboratory quality. A management review typically occurs once every 12 months (36).

- Change processes: Implementing a specific change in the laboratory is a linear process usually organized with the help of procedures such as project management and is restricted by time. In case of major change processes, it can be valuable to develop a structured communication plan. Gardner identified seven mind levers that interfere with the process of change and 
which must be opposed for the process of change to be linear: Reason, research, resonance, descriptions, resources and rewards, real-world events and resistance (37).

- Staff training: Organizational staff structure in genetic laboratories comprises clinicians, scientists and technicians who are expected to comply with the required standards and training with high priority (38).

IS0 15189 can be used by any authorized accreditation organizations and does not require re-accreditation if the laboratory changes its method of analysis. ISO 15189 allows other accreditation bodies to adapt ISO 15189 to local circumstance.

The ISO publishes the documents in multiple languages and copies of ISO standards can be obtained through the website of ISO (http://www.iso.org).

\section{Other sources of standards for the accreditation of genetic laboratories}

ISO is the main reference source for quality control of genetic laboratories, but subsequently other establishments for genetic laboratories have been developed, always referring to the ISO system and numerous guidelines have been published. For instance, OECD has published specific guidelines for quality assurance in molecular genetic testing, which might be used in combination with the existing accreditation standards for improvement of the system. These guidelines can be download from the OECD website (http://www.oecd.org).

In 2008, the Maputo Declaration on strengthening of laboratory systems recognized the following as areas requiring strengthening in resource-limited settings: leadership, human resources, career path structures, retention of staff, national laboratory policies, strategic planning, physical infrastructure, supply-chain management and quality management systems (39). Following the Maputo statement, the World Health Organization (WHO) denoted the relevance of laboratory quality management systems (40). In 2012, in line with the Health 2020 European policy for health and well-being, the WHO Regional Office for Europe (OMS) started the "Better Labs for Better Health" initiative (41). The "Better Labs for Better Health" approach indicate the minimum quality standards for laboratory as a requirement to provide accurate and reliable results in a timely manner for disease prevention and management (http://www.euro.who/). For each country, the first step towards "Better labs for better health" is the development of a national laboratory policy by a formally recognized national laboratory working group that must base on broad consensus and lead to the development of national strategic plans (42). Three new challenges are identified in "Better labs for better health program": accessibility of services, sustainable financing of laboratory activities and ethics. The policies identified the essential elements for strengthening the laboratory health systems: human resources management, infrastructure development and rationalization, sampling, supply-chain management and maintenance of laboratory equipment, management systems, laboratory information systems, biosafety and waste management $(40,43)$. Laboratory testing quality is necessary also in low- and middle-income countries (44). Ebola virus disease outbreak in West Africa provide an example of how functioning laboratories are in need for disease control and prevention (45). However, despite progress made, only about $30 \%$ of countries reported meeting the IHR core capacities' requirements for quality surveillance (46). Part of the global WHO 2008 Vision, according to the Maputo statement, can be performed on the implementation of national laboratory quality standards. The ISO 15189 covers a variety of clinical settings (?), genetic laboratories require more specific certification standards than the generic analysis laboratories. For example, in Italy genetic laboratories use ISO 15189 standards, but also use Sigucert certification. Copies of Sigucert can be download from the SIGU website (https://www.sigu.net). European Biotechnology association "EBTNA" through EBTNA lab offers a new certification that is more synthetic and essential than Sigucert but more suitable for a European system of genetic laboratories and it is easy to be accessed because it is clear, concise and intuitive. The Fig. 1 show a scheme of standards for the accreditation of genetic laboratories.

\section{EBTNA practice certification}

Main characteristics of European Biotechnology Thematic Network Association (EBTNA) is the presence of symbiotic relationship between science, education and biotechnological industry (47). The EBTNA has proposed a simple and efficient certification system according to IS0 15189 and to the model established by WHO which promoted the initiative "Better Labs for Better Health" dictating the minimum quality standards for laboratories as a requirement to provide reliable results in a timely manner for disease prevention and management. The EBTNA certification follows the EMQN model (https:// www.emqn.ora/eman/Home). To perform this service the lab has to be accredited by the International standard ISO 17043. ISO/IEC 17043:2010 specifies general requirements for the competence of providers of proficiency testing schemes and for the development and operation of proficiency testing schemes. Developing on the EMQN model, the EBTNA laboratory provide genotyped samples to laboratories enrolled by paying a fee to this quality assessment program to verify their operational standards. The EBTNA lab retrieve the results from all the participants to the programs and will evaluate the results obtained in order to give a quality score to each laboratory. In return this laboratory provides a quality certification. The EBTNA program follows the hub and spoke model, so there must be a connecting laboratory that meets a fixed quality standard. There are centralized genetics laboratories highly specialized for the diagnosis of genetic diseases known as "Hub centres" and specialized peripheral clinical structures that follow patients known as "Spoke centres". This will bring life to European network similar to the GENDIA model but with a certified standard of quality. GENDIA (http://www.GENDIA.eu/) is an international network consisting of more than 100 laboratories located in the USA, Europe, Asia and Australia, offering worldwide more than 3000 different genetic tests. Its' mission 


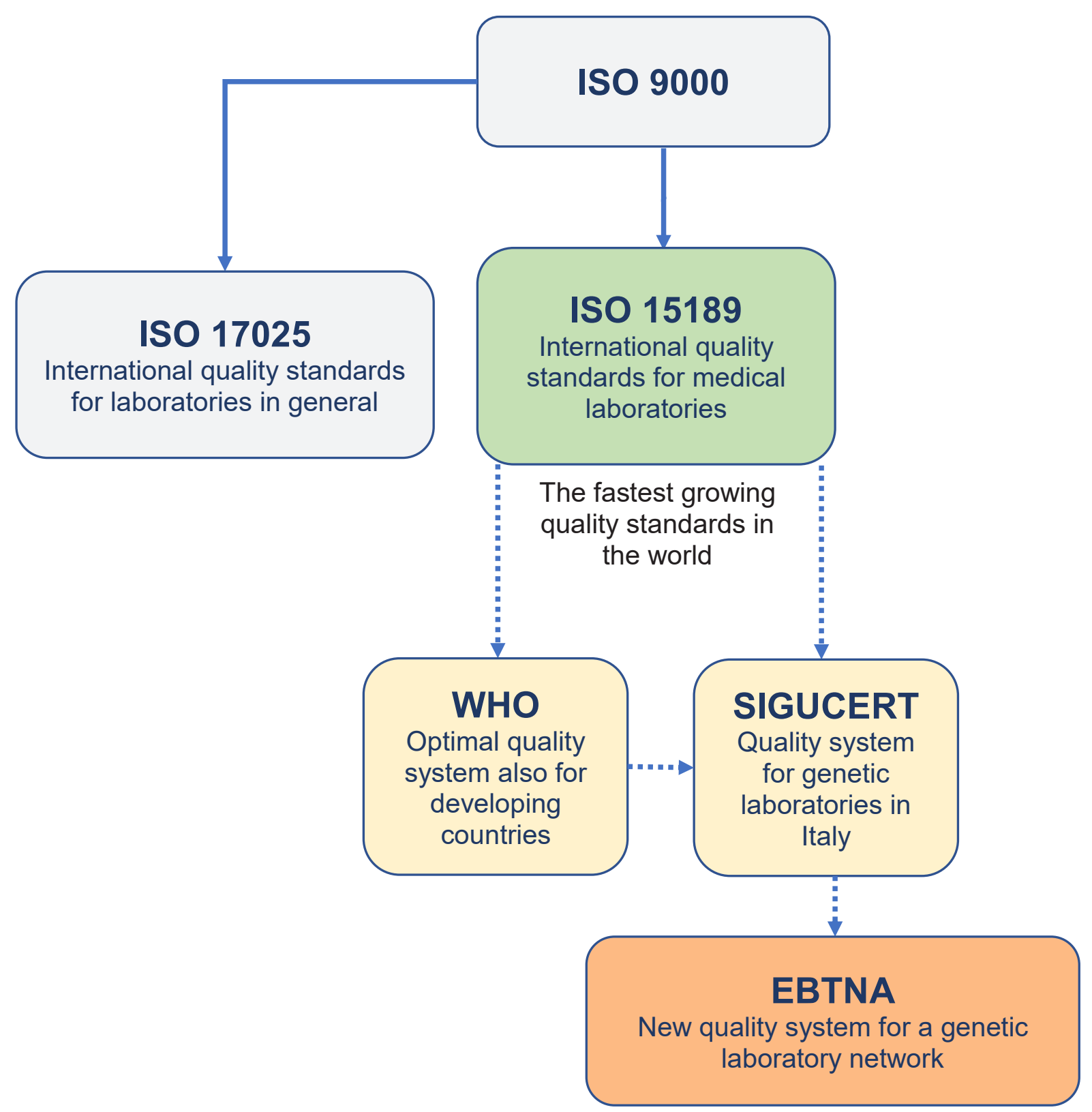

Figure 1. Standards for the accreditation of laboratories.

is to improve the accessibility, cost-effectiveness and quality of genetic diagnostics on a global scale. Such certification system of a laboratory network is important to improve the availability of genetic testing throughout the network members, which establishes a liaison across laboratories for mutual benefits with an economical growth to disadvantaged areas across Europe. Furthermore, it identifies good common practices following high quality standards.

Each laboratory within the network must perform selected genetic tests for all the members, so that the network as a whole could offer a vast majority of genetic tests with highest quality and lowest price. The network must cover all parts of Europe including regions that do not provide a wide range of diagnostic genetic test list. The network has to offer a good service to patients and ensure that each partner has economic gains. The EBTNA main laboratory has to be based in an area of Europe that has low rental cost and low taxes. Strengthening laboratory services includes several goals:

- Provide quality laboratory support for illnesses;

- Improve the quality of laboratory services in Europe for routine surveillance;

- Provide guidance on the most appropriate laboratory technologies and best practices;

- Effective transfer technology and sharing of knowledge;

- Promote the integration and / or coordination of specific laboratories for diseases with other laboratory activities;

- Attract, map resources and coordinate their mobilization to strengthen the network.

To implement the quality management system, activities must be divided into four stages of implementation:

- I) Ensure that the primary process of the lab works properly and safely; 


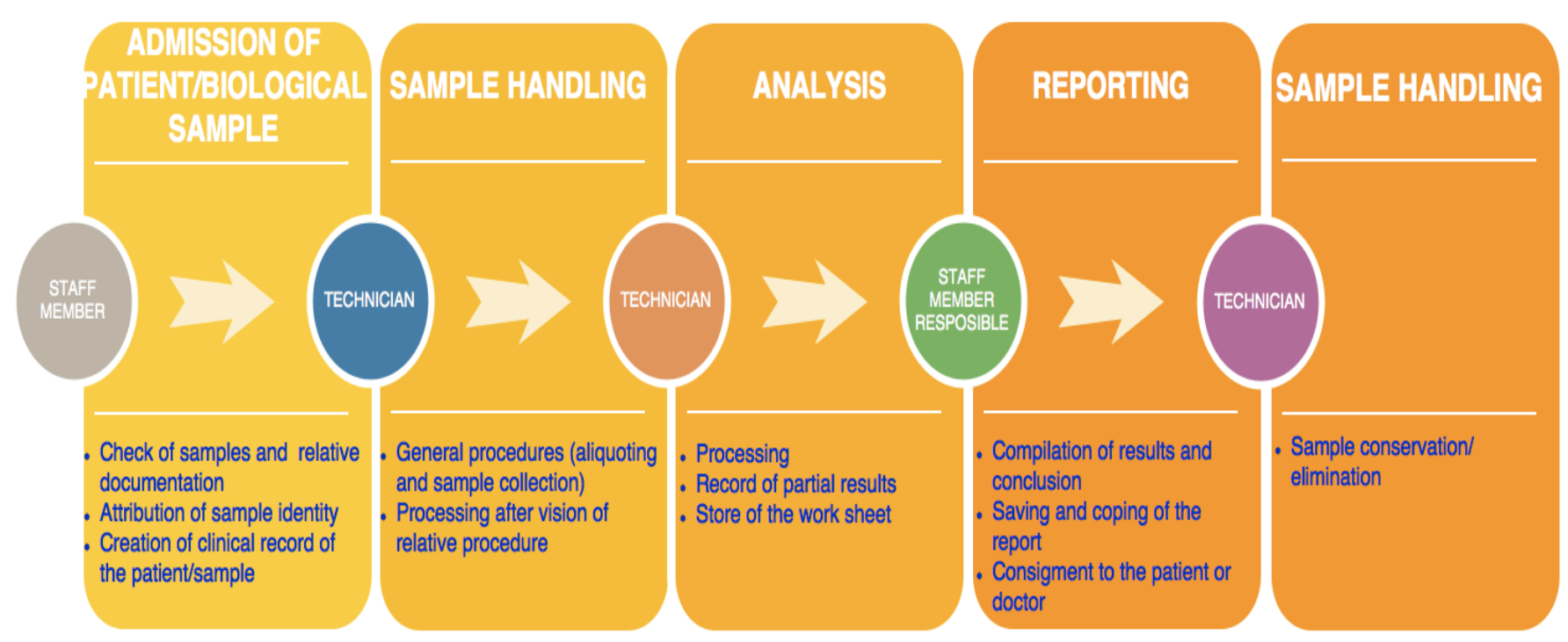

Figure 2. Scheme of the EBTNA quality system process.

- II) Check and ensure quality and create traceability;

- III) Ensure proper management leadership and organization;

- IV) Create a continuous improvement and prepare for accreditation.

If there is stringent application to standards, this has to be certified. It is indispensable to demonstrate that the genetic test satisfies the requirements by clinical and molecular guidelines. The conformity assessment process is advantageous for patients and clinicians, also it gives a competitive edge and helps regulators to ensure that safety conditions are met.

Key points of the quality control system implementation are:

- A trained and certified personnel;

- A set of documentations (procedures, manuals and protocols);

- A working system and a reporting system for management, when choosing an appropriate service based on effectiveness indicators, statistical accounting systems and monitoring of molecular genetic tests.

The products of a genetic laboratory are authorized reports containing laboratory testing results, as well as data on the patient (name, age, sex, and diagnosis), type of biological sample, time of taking it and delivering it to the laboratory, actual interpretation on of the results and other related information.

\section{Procedures, manuals and protocols of EBTNA quality assurance system.}

EBTNA quality assurance system is a simple application that can easily be accessible as a source of utility for those genetic laboratories willing to join the network. To ensure proper coherence to quality standards for genetic laboratories it is crucial that the set of documents including manuals, procedures, and protocols are prepared systematically and they are basic enough to provide homogeneity, clarity and completeness of the quality assurance process.

The manuals provide operational guidance on how to perform specific tasks such that the document illustrates what exactly the operator must do in order to ensure the quality of the system. The procedures describe the organizational and management modes so as to identify roles and responsibilities and to pursue the goals of effectiveness, efficiency and process control. The protocols are complex of rules and procedures to be followed for carrying out technical-professional activities. The EBTNA quality system organization is described in Fig 2.

EBTNA standards are given in the supplemental materials under the quality folder. Files with "PO" include planning and provision of molecular biology service (S1) and instructions for sending samples (S2) ; "admission" (S3); "working instructions" which includes extraction of DNA from blood Mini Kit, Gel electrophoresis, DNA quantification, PCR amplification of DNA and purification of PCR products (S4: a,b,c,d); " forms" which include informed consent, PCR amplification worksheet, report, worksheet restriction enzyme digestion, clinical record of patients, single sample sequencing request, genetic test request and sample receipt form (S5:a,b,c,d,e,f,g,h) and some other examples of protocols "protocols"(S6:a,b,c). Further information and details can be found on EBTNA's website (www.ebtna.net).

\section{Conclusions}

Clinical laboratory tests are mainly required for two reasons; one is to confirm diagnosis and the other is to monitor disease activity. Laboratory quality management covers diverse aspects of genetic testing including parameters such as internal and external quality assessments, control materials, training, document controls, maintenance of equipment, internal auditing and laboratory accreditation. The improvement in appropriate use of laboratory testing is required as it is an essential part of many health care systems (24). This concept has been described and the inter-relations are gathered in a pyramidal form in which the major phases of the evaluation are technical quality of the test, diagnostic accuracy, change in diagnostic thinking, change in patient management, change in patient outcomes and societal costs and benefits (48). At the heart of 
diagnostic effort is the foundation of a laboratory network, which can foster sharing of skills and knowledge to improve the diagnosis. Genetic laboratory networks must encompass many aspects, such as quality management, information databases, new technologies, public health and education. On this basis, EBTNA has developed a quality system based on international standards that can guarantee equal results by all laboratories affiliated by the network with the same efficiency and safety.

\section{References}

1. Kroese M, Zimmern RL, Sanderson S. Genetic tests and their evaluation: can we answer the key questions? Genet Med. 2004;6(6):475-80.

2. Kaul KL, Sabatini LM, Tsongalis GJ, Caliendo AM, Olsen RJ et al. The Case for Laboratory Developed Procedures: Quality and Positive Impact on Patient Care. Acad Pathol. 2017;16:4.

3. Hallworth MJ. The "70\% claim": What is the evidence base? Ann Clin Biochem 2011;48:487-8.

4. Badrick T. Evidence-based laboratory medicine.Clin Biochem Rev. 2013;34(2):43-6.

5. Aakre KM, Langlois MR, Watine J, Barth $\mathrm{JH}$, Baum H, et al. Critical review of laboratory investigations in clinical practice guidelines: proposals for the description of investigation. Clin Chem Lab Med. 2013; 51(6):1217-26.

6. Kirk CJ, Shult PA. Developing Laboratory Networks: A Practical Guide and Application Public Health Rep. 2010; 125(Suppl 2): 102-109.

7. Monach PA. Repeating tests: different roles in research studies and clinical medicine. Biomark Med. $2012 ; 6(5): 691-703$.

8. Hawkins R. Managing the pre- and post-analytical phases of the total testing process. Ann Lab Med. 2012;32(1):5-16.

9. ISO 3534-1;3.14"Statistics - Vocabulary and symbols part1: General statistics terms and terms used in probalility".

10. Karkaousos P, Evangeloupos A. In InTech, Applications and Experiences of Quality Control. Shanghai: Springer; 2011. Quality control in clinical laboratories; pp. 321-360.

11. Endrullat C, Glökler J, Franke P, Frohme M Standardization and quality management in next-generation sequencing.. Appl Transl Genom. 2016;1;10:2-9.

12. Aziz N, Zhao Q, Bry L, Driscoll DK, Funke B, et al.College of American Pathologists' laboratory standards for next-generation sequencing clinical tests. Arch Pathol Lab Med. 2015;139(4):481-93.

13. Badrick T. The quality control system. Clin Biochem Rev. 2008;29 Suppl 1:S67-70.

14. Westgard JO. Internal quality control: planning and implementation strategies. Ann Clin Biochem 2003;40:593-611.

15. External quality assessment of health laboratories: Report on a WHO Working Group, 1981.

16. Cembrowski GS, Carey RN. Adding value to proficiency testing programs. Clin Chem 2000;46:7-8.

17. Berwouts S, Fanning K, Morris MA, Barton DE, Dequeker E. Quality assurance practices in Europe: a survey of molecular genetic testing laboratories. Eur J Hum Genet. 2012;20(11):1118-26.

18. Ezzelle J, Rodriguez-Chavez IR, Darden JM, Stirewalt M, Kunwar $\mathrm{N}$, et al. Guidelines on good clinical laboratory practice: bridging operations between research and clinical research laboratories. Pharm Biomed Anal. 2008;46(1):18-29.

19. De la Salle B, Meijer P, Thomas A, Simundic AM. Special issue on External Quality Assessment in Laboratory Medicine - current challenges and future trends.Biochem Med. 2017;27(1):19-22.

20. Kristoffersson U, Schmidtke J, Cassiman JJ, Dequeker E. Quality management systems and accreditation; in Quality Issues in Clinical Genetics Services. Springer: New York, 2010.
21. International Organization for Standardization: ISO/IEC 17025 general requirements for the competence of testing and calibration laboratories, 2005.

22. Schneider F, Maurer C, Friedberg RC. International Organization for Standardization (ISO) 15189. Ann Lab Med. 2017;37(5):365370.

23. Guzel O, Guner El. ISO 15189 accreditation: Requirements for quality and competence of medical laboratories, experience of a laboratory I. Clin Biochem.2009;42(4-5):274-8.

24. Berwouts S, Morris MA, Dequeker E. Approaches to quality management and accreditation in a genetic testing laboratory. Eur J Hum Genet. 2010;18 Suppl 1:S1-19.

25. R. Selvakumar. Good Laboratory Practices Indian J Clin Biochem. 2010; 25(3): 221-224.

26. Mattocks CJ, Morris MA, Matthijs G, Swinnen E, Corveleyn A, et al. A standardized framework for the validation and verification of clinical molecular genetic tests.; Eur J Hum Genet. 2010;18(12):127688.

27. Cassiman JJ. Research network: EuroGentest - a European Network of Excellence aimed at harmonizing genetic testing services. Eur J Hum Genet. 2005;13:1103-1105.

28. Kalman LV, Datta V, Williams M, Zook JM, Salit ML, Han JY. Development and Characterization of Reference Materials for Genetic Testing: Focus on Public Partnerships. Ann Lab Med. 2016;36(6):513-20.

29. Directive $98 / 79 / E C$ of the European Parliament and of the Council of 27 October 1998 on in vitro diagnostic medical devices. Official J. L 331, 1-37 (1998).

30. Burnett $\mathrm{D}$ : A practical guide to accreditation in laboratory medicine, 2002.

31. Hwang SH, Jung SK, Kang SJ, Cha HS, Chung SH, Lee DH. Development of a document management system for the standardization of clinical laboratory documents. Ann Lab Med. 2013;33(6):441-8.

32. Westgard JO. Internal quality control: planning and implementation strategies. Ann Clin Biochem 2003;40:593-611.

33. Deming WE: Out of the crisis, 2000.

34. Ho B, Ho E. The most common nonconformities encountered during the assessments of medical laboratories in Hong Kong using ISO 15189 as accreditation criteria. Biochem Med. 2012;22(2):24757.

35. European cooperation for Accreditation of Laboratories: EAL-G3 internal audits and management reviews for laboratories, 1996.

36. Balague N and Saarti J. Managing Your Library and Its Quality: The ISO 9001 Way. Oxford, UK: Chandos Publishing; 2011.

37. Gardner H. Changing minds: the art and science of changing our own and other people's minds, 2006.

38. Walz SE. Education and training in laboratory medicine in the United States. JIFCC. 2013;24(1):1-3.

39. The Maputo Declaration on strengthening of laboratory systems. Brazzaville: WHO Regional Office for Africa; (htp://www.who.int/ diagnostics_laboratory/Maputo-Declaration_2008.pdf, accessed 20 June 2015). The Maputo Declaration on strengthening of laboratory systems. Brazzaville: WHO Regional Office for Africa;2008 (http://www.who.int/diagnostics_laboratory/Maputo-Declaration_2008.pdf, accessed 20 June 2015).

40. Joint $\mathrm{WHO}-\mathrm{CDC}$ conference on health laboratory quality systems. Geneva: World Health Organization; 2008 (http://www.who.int/ csr/ihr/lyon/report20080409.pdf?ua=1, accessed 6 August 2015).

41. Health 2020: the European policy for health and wellbeing [website]. Copenhagen: WHO Regional Office for Europe; htp://www.euro.who.int/en/health-topics/ health-policy/ health-2020-the-european-policy-forhealth-and-well-being,accessed 6 August 2015).

42. Brown CS, Zwetyenga J, Berdieva M, Volkova T, Cojocaru R, et al. New policy-formulation methodology paves the way for sus- 
tainable laboratory systems in Europe. Public Health Panorama. 2015;1(1):41-47.

43. Nkengasong JN, Nsubuga P, Nwanyanwu O, GershyDamet $\mathrm{GM}$, RoscignoG, et al. Laboratory systems and services are critical in global health: time to end the neglect? Am J Clin Pathol. 2010;134(3):368-73.

44. Olmsted SS, Moore M, Meili RC, Duber HC, Wasserman J, et al. Strengthening laboratory systems in resource-limited settings. Am J Clin Pathol. 2010;134(3):374-80.

45. Goodfellow I, Reusken C, Koopmans M. Laboratory support during and after the Ebola virus endgame: towards a sustained laboratory infrastructure. Euro Surveill. 2015;20(12):21074.
46. Katz R, Sorrell EM, Kornblet SA, Fischer JE. Global health security agenda and the international health regulations: moving forward. Biosecur Bioterror. 2014;12(5):231-8.

47. Bruschi F, Dundar M, Gahan PB, Gartland K, Szente M, et al..Biotechnology worldwide and the 'European Biotechnology Thematic Network' Association (EBTNA).Curr Opin Biotechnol. 2011;22 Suppl 1:S7-14.

48. Price CP. Evidence-based laboratory medicine: supporting decision-making. Clin Chem. 2000;46:1041-50. 